\title{
Thermal Influence Occurring Deviations in the Parameters of the Laser Beam Propagating through Atmospheric Air
}

\author{
Omar B. Yousef \\ University of Nyala, Faculty of Education, Department of Physics
}

\begin{abstract}
This study is aimed to determine deviations in the parameters of the laser beam which were caused by heat. In addition, the initial parameters of the laser beam before the effect of heat comparing with the parameters determined after the heat effect. As well as, based on the fluctuations occur in the parameters of the laser beam, the refractive index structure, inner scale, outer scale, and atmospheric coherence have been determined. By considering the laser beam propagates through atmospheric turbulence experience random changes, the propagation characteristic of the laser has been determined. The setup of experiment has been designed to make laser beam propagation through cross- section of turbulence air. The experiment took place in dark room and was structurally closed off from the outside environment. The results have been analyzed by both of origin program software (version-8), and excel software program.
\end{abstract}

Keywords: the refractive index structure, atmospheric coherence diameter, Gaussian function

\section{Introduction}

The interest in the spatially coherent beam as a tool improving the performance of laser communication systems was indicated recently in a number of publication $[11,10]$. Studies of the propagation of a partially coherent beam wave through atmosphere turbulence have been conducted over the past three decades by numerous researchers[ 3,4 ]. The important generalization that our approach provides is the ability to utilize any known atmospheric spectrum models [5] In this study, the heat indirectly effect on parameters of the laser beam specifically in both of the beam intensity and spot size. This effect makes the laser beam to be partially coherent. We consider the thermal turbulence model forms vertically large random numbers of windows air on path of laser propagation. These random windows scattering or diffraction the beam and finally originates fringes ( bright or dark ) which can see in monitor computer. In this study, ( for case of fluctuations in parameters of the laser beam, we restrict our works in the Klmogorov spectrum model to satisfy the purpose.

Turbulence can be described as the random mixing of air particles in the atmosphere due to either rapid or small-scale spatial and temporal refractive index fluctuations in temperature $[3,6]$.

Theories relating to atmospheric turbulence have been studied over many decades in order to better understand the impact of turbulence on the propagation of a laser beam through the atmosphere[6]. Turbulence can be described as the random mixing of air particles in the atmosphere due to either rapid or small-scale spatial and temporal refractive index fluctuations in temperature [3.4].Although slight variations in temperature can cause changes in the refractive index of air ( of the order 0.1-1.0K), the accumulative effect of such inhomogeneities expanding over vast distances poses significant challenges for laser beam propagation[7]. Research has shown that refractive index fluctuations of the atmosphere are significant near the surface of the earth and negligible at higher altitudes [4]. These refractive index fluctuations causes random phase perturbations of the laser beam that can lead to beam distortion [ 13]. In addition, laser propagation through turbulent media can result in scintillation beam wander [ 14] and beam spreading [11] The extent to which these factors affect the beam depend largely on the varying nature of the turbulent eddies that exist at several altitudes. Knowledge oftheses effects, attained over the years, has been used notably in the domains of military [9], radar [11], remote sensing [10], satellite communications [8] and medical diagnostics [12] ,[15]. The turbulent region exhibited very strong disturbance, in range of ( $\left.1.1 \times 10^{-12} m^{-2 / a}-2.7 \times 10^{-12} m^{-2 / 2}\right)$, ( Shivan M. 2014 ) .

In previous study prepared by Shivan M. ( 2014 ), unique modification have been applied to an existing system to measure the effects of thermal turbulence on a laser beam. In this study, the generation of different types of lasers using integrated circuit (555 timer) with mode called monostable circuit. After that passed each pattern through a medium effected by the heat, and calculated variables based on the Gaussian function, and these values were compared to the fundamental values of the beam before it passes in the turbulence medium

In lab, we passed the laser beam over the flame, to identify the effects of the thermal turbulence on the laser beam propagation through air as illustrated in Figure(2). The spectrometer ( USB2000 Ocean ), and CCD detector have been used to analyze the patterns of the laser beam passed over the flame at constant pressure. This technique may be fully quantifying the thermal influence on laser beam propagation through atmospheric air. The fluctuations in the parameters of the laser beam caused by heat were estimated as follow: the intensity of the beam is ranged between ( $24 \mathrm{~dB}-32.3 \mathrm{~dB})$, the wavelength is ranged between $(684.5 \mathrm{~nm}$ $-1276.3 \mathrm{~nm}$ ), the amplitude is ranged between ( $5 \mathrm{~m}-27.4 \mathrm{~m}$ ), and divergence of the beam is ranged between $\left(9.6^{\circ}\right.$ 


\section{International Journal of Science and Research (IJSR) \\ ISSN (Online): 2319-7064}

Index Copernicus Value (2013): 6.14 | Impact Factor (2015): 6.391

$\left.17.9^{\circ}\right)$. Also, the parameters relevant with random fluctuations that caused by heat in pathway of the laser beam were found as follow: The range of the atmospheric coherence diameter is ranged between: $r_{0}$ ( $\left.2 \times 10^{-28} m^{2 / a}-5 \times 10^{-4} m^{2 / a}\right)$, the refractive index structure function $C_{n}^{2}$

$\left.16 \times 10^{-21} m^{-2 / a}-1.2 \times 10^{-12} m^{-2 / a}\right)$, the inner scale $i_{0}$ $\left(1.8 \times 10^{-5} m^{2 / a}-7.4 \times 10^{-5} m^{2 / a}\right)$, and the outer scale $l_{0}\left(0.001 m^{2 / a}-0.008 m^{2 / a}\right)$. Previous amounts represent a good description of fluctuations experience the laser beam as it passes through inhomogeneous medium, where they depends on two- third law that is derived by Klmogorov.

\section{Theory}

Theoretically, the random fluctuations in the refractive index of the atmosphere alter the propagation pathway of light beams, which in turn effects their initial phase fronts. Once light propagates through a turbulent atmosphere, the phase fronts become distorted and experience random changes in the beam direction ( beam wander) as well as random intensity fluctuations ( scintillation ) [14]. A good measure of turbulence is the refractive index structure coefficient $C_{n}^{2}$. Because the air needs time to heat up, the turbulence is typically greatest in the middle of the afternoon ( $\left.C_{\mathrm{n}}^{2}=10^{-1 a} m^{-2 / a}\right)$ and weakest an hour after sunrise or sunset $\left(C_{n}^{2}=10^{-17} \mathrm{~m}^{-2 / 2 / 2}\right), C_{n}^{2}$ is usually largest near the ground, decreasing with altitude,[1]. The key to obtaining information about the way in which beams are affected by turbulence is to determine the refractive index structure coefficient $C_{n}^{2}$ given by [4]

Where,

$$
C_{n}^{2}=79 \times 10^{-6}\left(\frac{P}{T^{2}}\right) C_{T}^{2}
$$

$$
C_{T}^{2}=\sqrt{\left\langle\left(T_{1}-T_{2}\right)^{2}\right\rangle} T^{-1 / 2}
$$

where $p$ is the atmospheric pressure in millibars and $<>$ represents the ensemble average. $C_{T}^{2}$ is a measurable quantity defined by temperature differences $T_{1}$ and $T_{2}$, between two reference points separated by a distance $r$.

Particular emphasis is placed on the phase structure function as it represents a measure of the phase correlation between two points in a plane traversing perpendicularly to the direction of propagation. The phase structure function $D_{s}$ and atmospheric coherence diameter $r_{0}$ are given by [2] and [5]

$$
D_{s}=2.91 C_{n}^{2}\left(1-0.8\left[\frac{2 \pi y}{L_{0}}\right]^{\frac{1}{3}}\right.
$$

And

$$
r_{0}=\left[\left(\frac{2 \pi}{\lambda}\right)^{2} \int_{z_{1}}^{z_{2}} C_{n}^{2} d z\right]^{2 / a}
$$

Atmospheric turbulence arises from random inhomogeneities ( eddies ) of the refractive index in the atmosphere. As it follows from the electromagnetic wave propagation theory the optical wave are significant impacted by these refractive index fluctuations. These fluctuations are generally considered random fields of both space and time variables.

The basis for theoretical and experimental studies of atmospheric turbulence is classical Kolmogrov turbulence theory. $[1-3,4]$. This theory describes atmospheric optical turbulence dynamics inside so- called inertial subrange that is defined by inner scale $i_{0}$, and outer scale $L_{0}$ sizes of turbulence eddies [4]. The Kolmogorov turbulence theory operates on the assumption that the random fields of refractive index inhomogeneities are stability homogeneous and isotropic. In the Kolmogrov turbulence theory, the strength of turbulence is characterized by the refractive index structure parameters $C_{n}^{2}$. The magnitude of $C_{n}^{2}$ is typically used to distinguish between three turbulence regimes: the weak $\left(C_{\mathrm{n}}^{2} \leq 10^{-17} \mathrm{~m}^{-2 / a}\right)$, moderate ( $\left.10^{-17} \leq C_{n}^{2} \leq 10^{-14} m^{-2 / a}\right), \quad$ and strong $\left.C_{n}^{2} \geq 10^{-14} m^{-2 / a}\right)$ turbulence regimes $[4,10,11]$.

\section{Method and Materials}

The apparatus that have been used for conducting the experiment are: Diode laser (DL), timer model NE/555, capacitance $C_{e x}=0.1 \mu F$, resistance $R_{e x}=1 \mathrm{k} \Omega$, load resistance $\mathrm{R}_{\mathrm{L}}$, switching power supply Model SNG 1000 input $(230 \mathrm{~V}-50 \mathrm{~Hz}-20 \mathrm{~W})$ output $(3 \mathrm{~V}-4 \mathrm{~V}-5 \mathrm{~V}-6 \mathrm{~V}-7.5 \mathrm{~V}-9 \mathrm{~V}-$ $12 \mathrm{~V}(1.5 \mathrm{~V}-12 \mathrm{~V}$ ), ohmmeter, Spectrometer ( UB 2E7524 ), ADC type (USB 2000), CCD detector, Automatic heater, thermocouple model TC4M-14R, Computer. The experiment conducting with conditions are: room temperature $300 \mathrm{k}^{0}$, air pressure 0.014 mbar. Firstly, to operate diode laser the electric circuit has been connected as illustrate in Fig.(1).

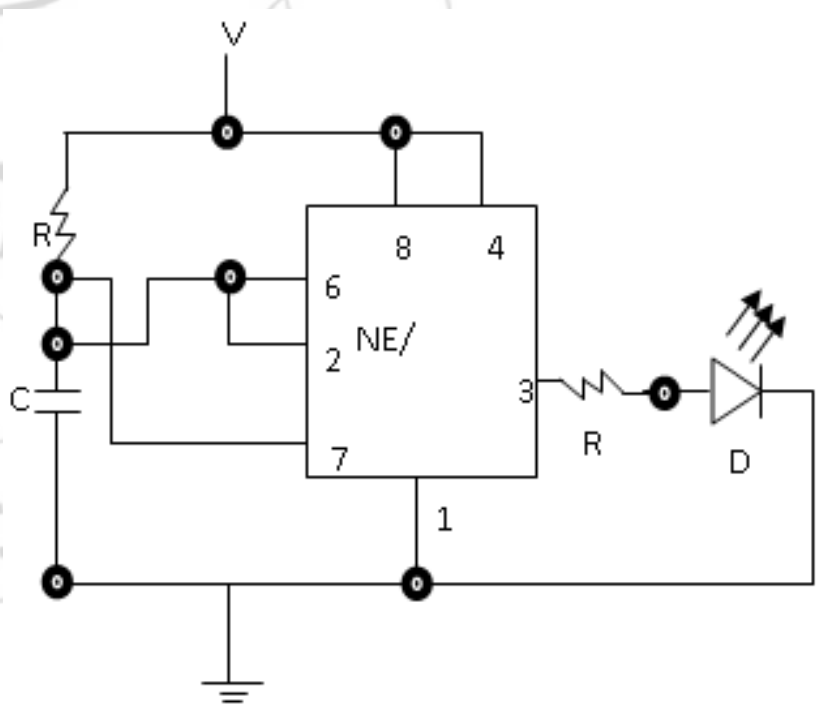

Figure 1: Monostable circuitoperating diode laser. 


\section{International Journal of Science and Research (IJSR) \\ ISSN (Online): 2319-7064}

Index Copernicus Value (2013): 6.14 | Impact Factor (2015): 6.391

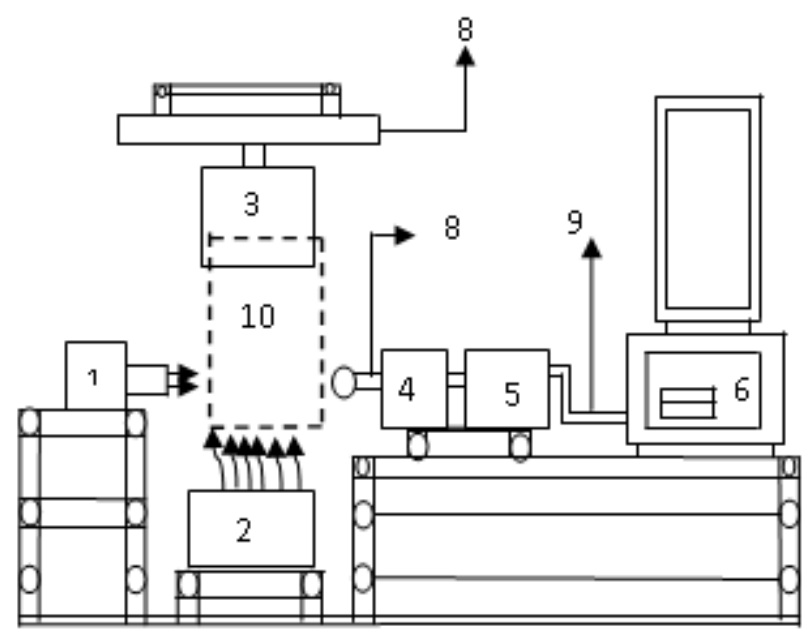

Figure 3: Complete Experiment Setup

Where: 1- Laser source , 2- Automatic heater,3Thermocouple 4- Spectrometer, 5- Photodetector, 6Computer, 8- Latch, 9- optical fiber cable, 10- USB cable, 11- Turbulence model.

\section{Experiment Description}

Figure. 3 illustrated a complete diagram of experiment setup, where the thermocouple used to generate turbulence through pathway of the laser propagation. By considering all optical components of the experiment are collimated, the laser beam directed toward optical fiber across the path of the diffusion heat. The experiment took place in dark room and was structurally closed off from the outside environment as there were no windows outlets.

Thermocouple located vertically above the optical path, so as to sense heat, then it can approach to measure temperature for several points along optical pathway by moving latch left to right. After short interval the air molecules which existence in distance between laser source and photodetector were heated. This process generates turbulent in optical pathway, the motion of air molecules be randomly. The laserbam propagating through inhomogeneous medium experience random changes in its parameters such as: direction, intensity, amplitude, wavelength, and energy. The temperature caused fluctuations in the main parameters which are relevant with atmospheric air, such as: refractive index structure function, atmospheric coherence diameter, inner scale, and outer scale. The most formulas that used to determine these parameters are semi- empirical formulas, such formula derived by [4]. In addition, the temperature caused fluctuations in the parameters that relevant with self characteristics of the laser beam, such as: spot size, waist, divergence angle, Rayleigh range, radius of curvature, and depth of focus of the beam. These parameters are determined by considering the Gaussian beam as symmetrical function.

\section{Data Analysis and Discussion}

The data were analyzed in a dark room that isolated from the external environment at a temperature $\left(300 \mathrm{k}^{\circ}\right)$ and atmospheric pressure $(0.014 \mathrm{mbar})$.

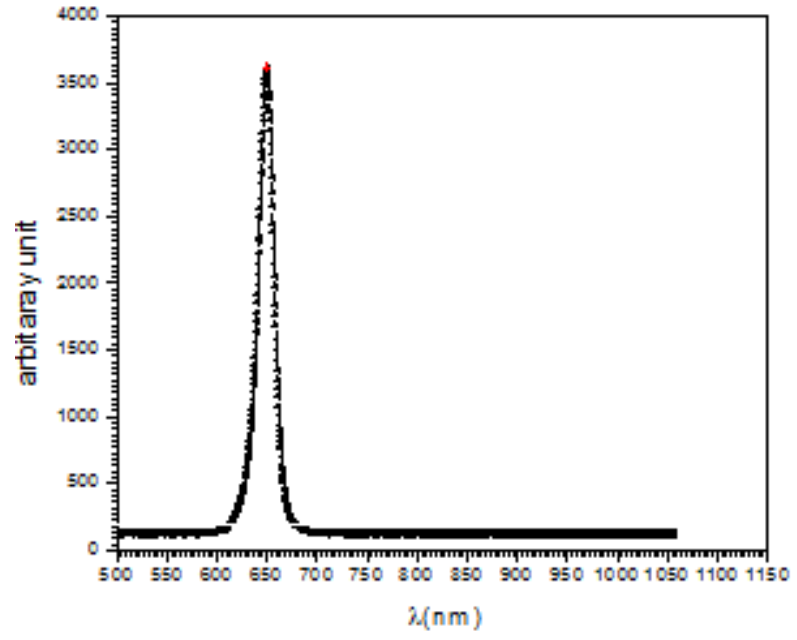

Figure 3: Pattern of the laser beam before thermal influence, $\mathrm{V}_{\mathrm{cc}}=4.5 \mathrm{~V}$

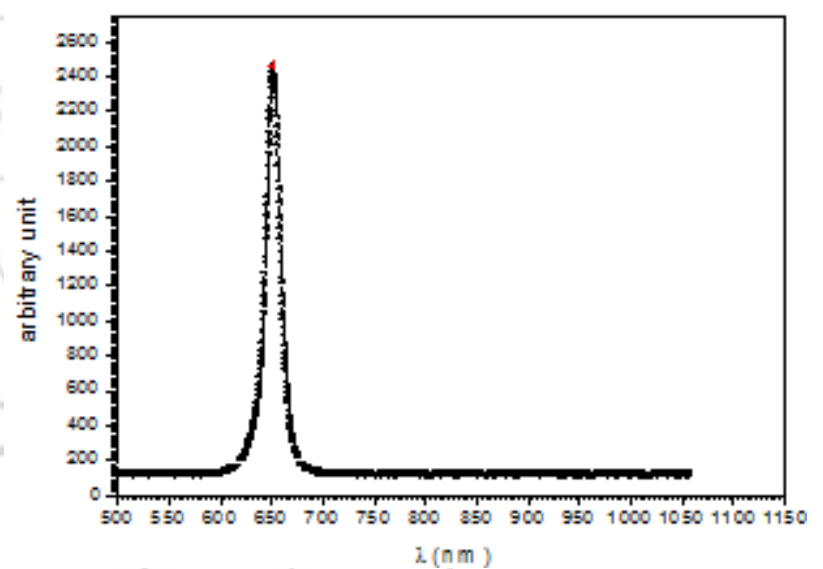

Figure 4: Pattern of the laser beam before thermal influence,

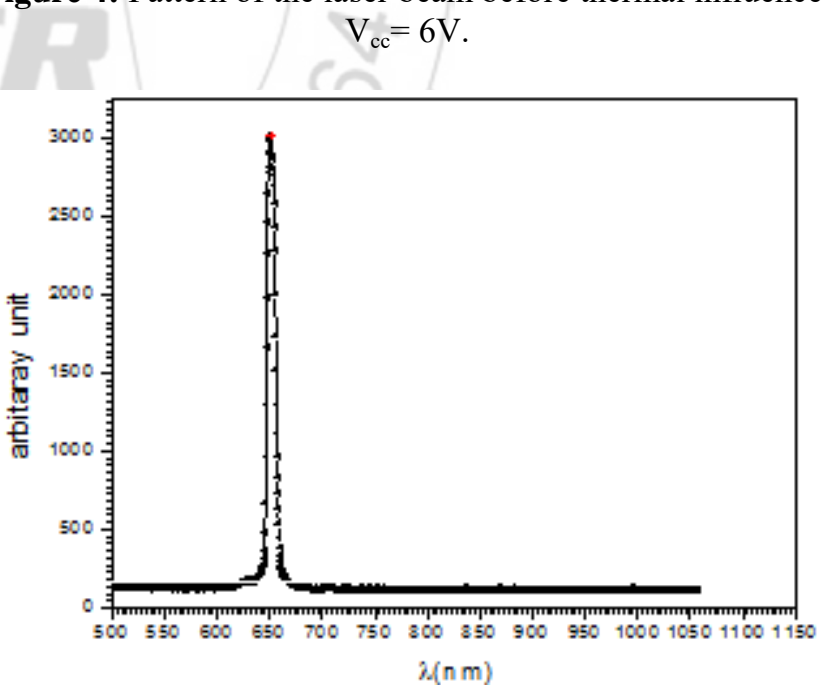

Figure 5: Pattern of the laser beam before thermal influence, $\mathrm{V}_{\mathrm{cc}}=7.5 \mathrm{~V}$ 


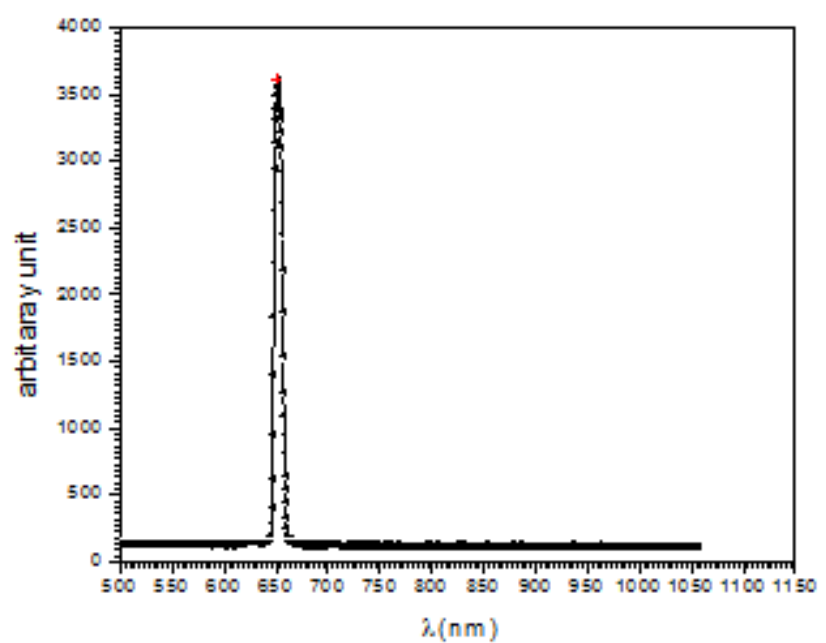

Figure 6: Pattern of the laser beam before thermal influence, $\mathrm{V}_{\mathrm{cc}}=9 \mathrm{~V}$.

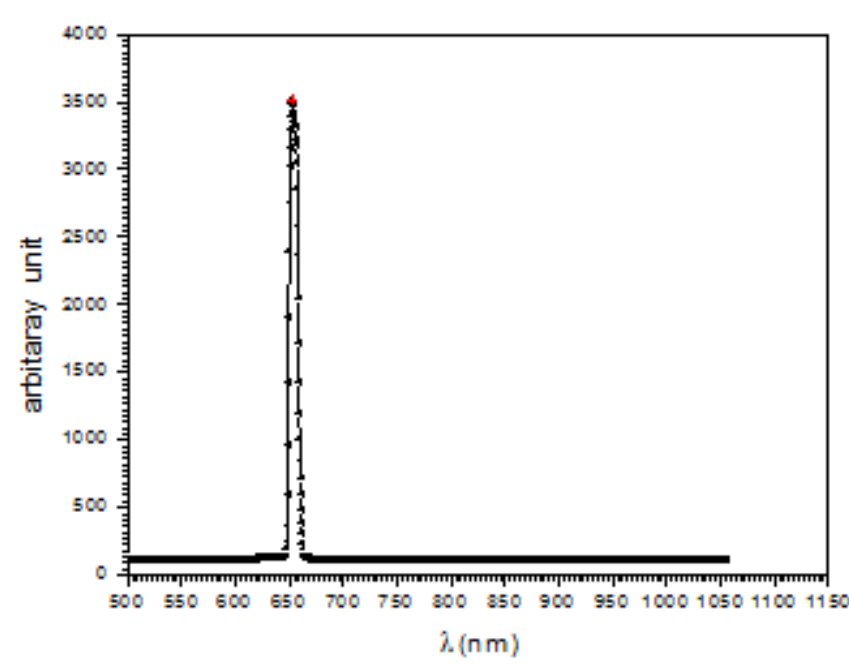

Figure 7: Pattern of the laser beam before thermal influence,

$$
\mathrm{V}_{\mathrm{cc}}=12 \mathrm{~V} \text {. }
$$

The figures from (3) to ( 7 ), shows the basic patterns of the laser beam before being influenced by heat, as each pattern depends on the amount of voltage applied on monostable circuit.

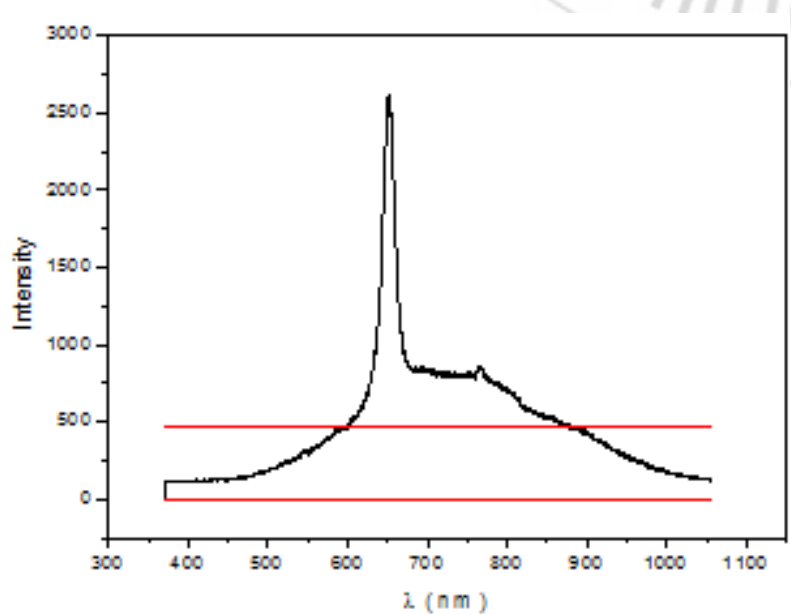

Figure 8: Pattern of the laser beam after thermal influence,

$$
\mathrm{V}_{\mathrm{cc}}=8.6 \mathrm{~V} \text {. }
$$

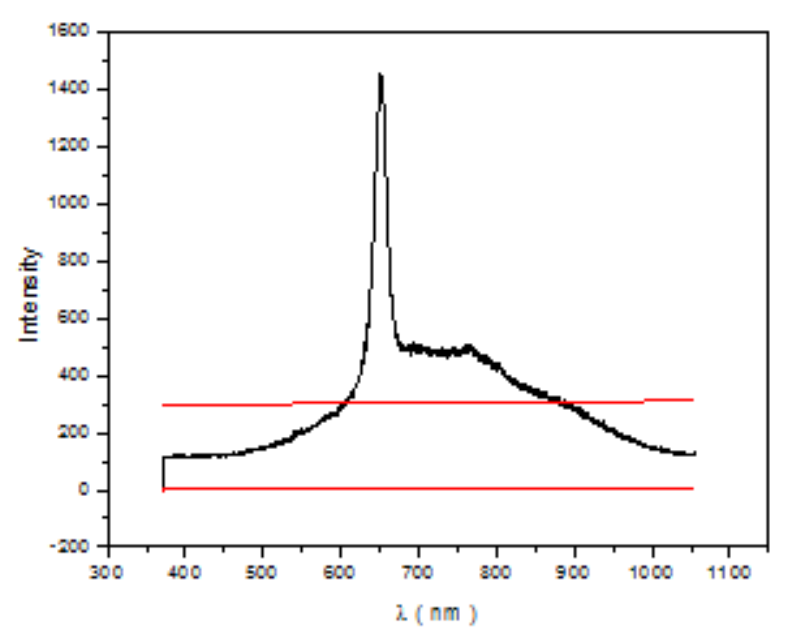

Figure 9: Pattern of the laser beam after thermal influence, $\mathrm{V}_{\mathrm{cc}}=5.1 \mathrm{~V}$.

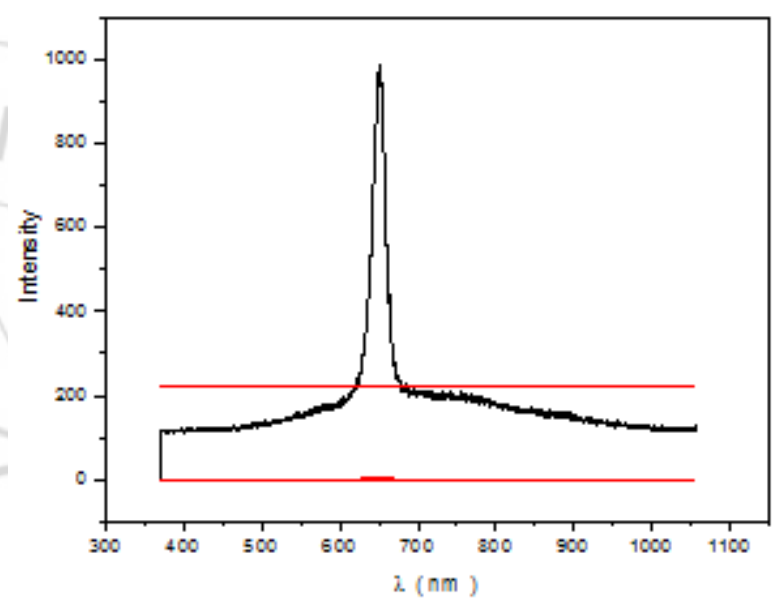

Figure 10: Pattern of the laser beam after thermal influence, $\mathrm{V}_{\mathrm{cc}}=3.4 \mathrm{~V}$.

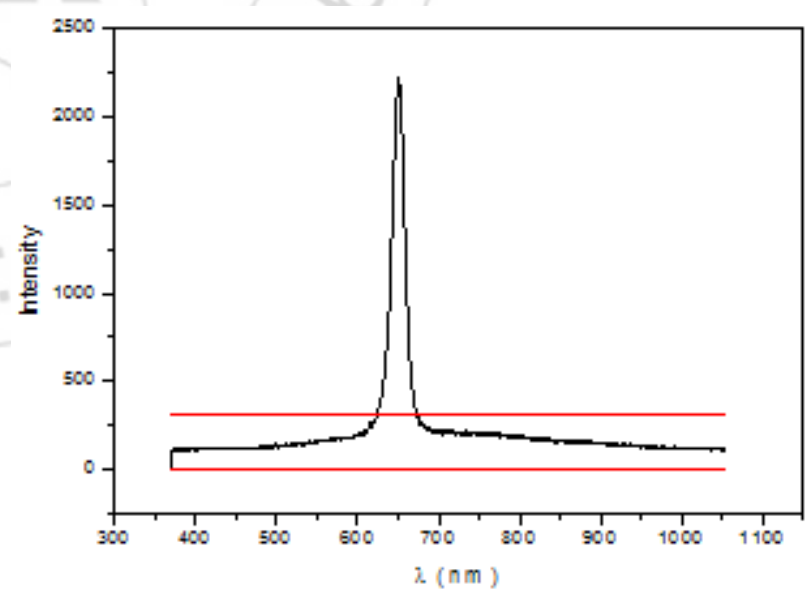

Figure 11: Pattern of the laser beam after thermal influence, $\mathrm{V}_{\mathrm{cc}}=7.7 \mathrm{~V}$. 


\section{International Journal of Science and Research (IJSR) \\ ISSN (Online): 2319-7064}

Index Copernicus Value (2013): 6.14 | Impact Factor (2015): 6.391

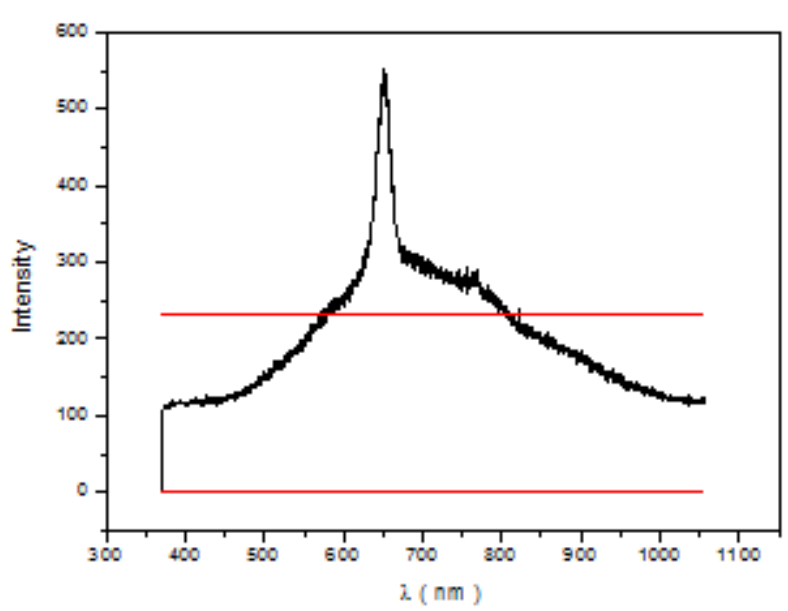

Figure 12: Pattern of the laser beam after thermal influence, $\mathrm{V}_{\mathrm{cc}}=2.05 \mathrm{~V}$.

Figures from ( 8 ) to ( 12) represent a laser patterns that passes through the hot air, these patterns reflect deviations laser variables values for their amounts core by thermal effect on the beam path. These patterns indicate to what extent the laser beam deflected when it passes through the center of a heterogeneous molecules moving randomly. For a pure Gaussian beam, $M^{2}=1$, where $\mathrm{M}^{2}$ is the propagation constant which is an accurate indication of the propagation characteristics of the beam. By comparing $M^{2}=1$ with values of $\mathrm{M}^{2}$ that resulted by magnitude of both of far field divergence $\theta_{R}$ and the beam waist $w_{O R}$, we found that the values of $\mathrm{M}^{2}$ for all patterns in figures from (8) to (12) are close to one or $M^{2} \approx 0.82$. This indicated that the beam propagationthrough inhomogeneous mediumit becomes not a pure Gaussian beam.

Table 1: Illustrated basic parameters of the laser beam, before thermal influence.

\begin{tabular}{|c|c|}
\hline parameter & value \\
\hline Wavelength $(\mathbf{n m})$ & 650.17 \\
\hline Intensity $(\mathbf{d B})$ & 35.4 \\
\hline Amplitude $(\mathbf{m})$ & 29.6 \\
\hline Beam divergence $(\mathbf{m}$ degree $)$ & 18 \\
\hline Waist beam $W_{\mathbb{D}}(\boldsymbol{\mu r a d})$ & 11.33 \\
\hline
\end{tabular}

By Comparing the results of table (1) with the table (2) we note a significant difference happen in the wavelengths of the patterns of the beam passing through turbulence medium and this is due to lack in propagation constant.

Table 2: shows the parameters of the laser beam, after thermal influence

\begin{tabular}{|c|c|c|c|c|}
\hline $\boldsymbol{A}$ & $\boldsymbol{I}$ & $\boldsymbol{\lambda}(\mathbf{n m})$ & $\boldsymbol{\theta}_{\text {OF }}(\mathbf{m}$ degree $)$ & $\boldsymbol{w}_{\text {OR }}(\boldsymbol{\mu r a d})$ \\
\hline $\mathbf{2 7 . 4}$ & 32.3 & 753 & 17.9 & 9.3 \\
\hline $\mathbf{1 1 . 4}$ & 31.6 & 753 & 17.9 & 9.5 \\
\hline $\mathbf{1 4 . 3}$ & 28.9 & 740 & 17.8 & 9.4 \\
\hline $\mathbf{5 . 7}$ & 32.8 & 7715 & 9.16 & 0.018 \\
\hline $\mathbf{2 7}$ & 24.5 & 664 & 16.9 & 9.9 \\
\hline $\mathbf{1 9 . 8}$ & 29.7 & 716 & 17.6 & 9.6 \\
\hline
\end{tabular}

Key: $A(m)$ amplitude, $I(d B)$ intensity

From table (2), we note that the lower energy of the beam occurs at large wavelength as illustrated in fig(13), this corresponds a far field divergence $\theta_{O R}=9.16$ mdegree, , $w_{\text {OR }}=0.018 \mu \mathrm{rad}, M^{2} \ll 1$. At the same time, we note many deviation occurs when the beam wavelength (7715 $\mathrm{nm})$ and then happened a significant reduction in the propagation constant which is arrived $M^{2}=8.08 \times 10^{-4}$, this values corresponds to the far field beam waist that equal $w_{O R}=0.018$ rrad.

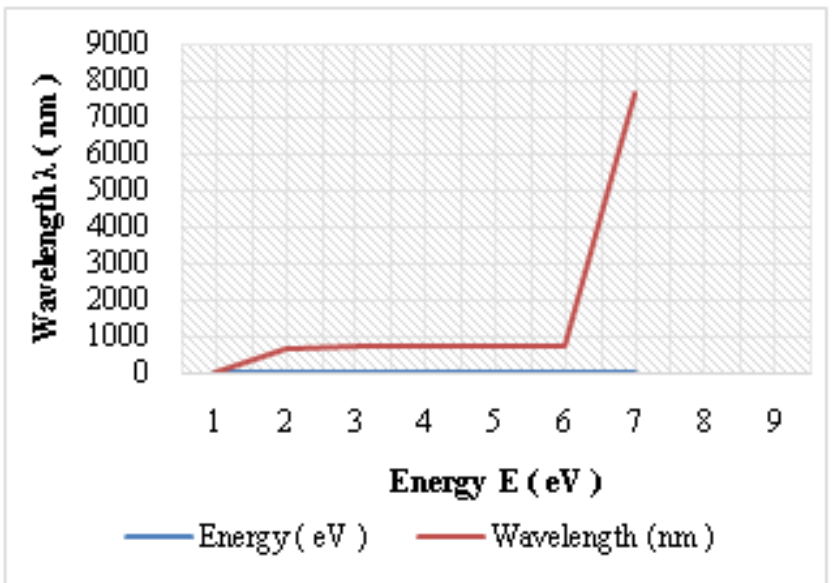

Figure 13: decreasing Energy E (ev ) relative to wavelength $\lambda(\mathrm{nm})$.

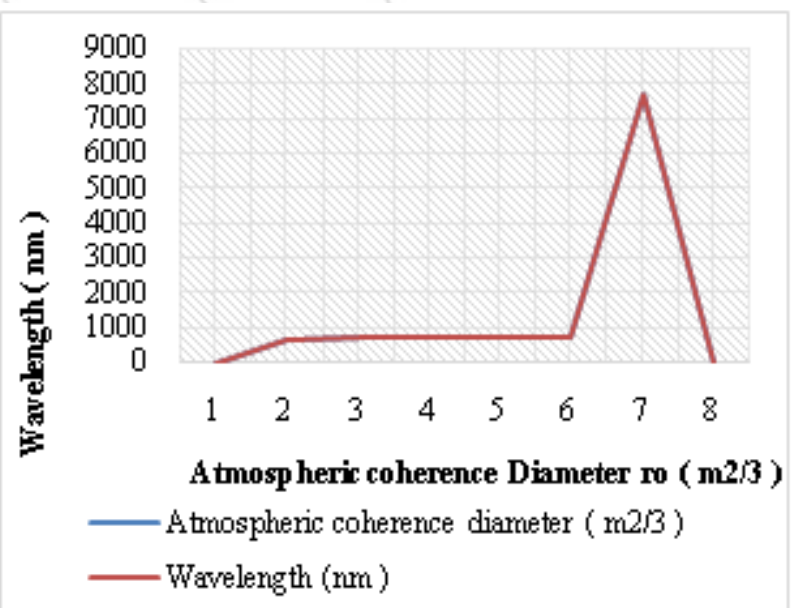

Figure 14: Shows relation between Wavelength $\lambda(\mathrm{nm})$ and Atmospheric coherence diameter $r_{0}\left(\mathrm{~m}^{2 / a}\right)$.

Figure (14) shows, the atmospheric coherence diameter $r_{0}$ is inversely proportional to wavelength. According to eq( 3 ), we note that the atmospheric coherence diameter reaches a maximum value $\left(5 \times 10^{-4} \mathrm{~m}^{2 / a}\right.$ at a point far away $(\mathrm{z}=$ $6 \mathrm{~mm}$ ) from the origin point, in contrast, we find that its lowest value of $r_{0}\left(2 \times 10^{-28} \mathrm{~m}^{2 / a}\right)$ this corresponds a maximum wavelength ( $7715 \mathrm{~nm}$ ), strength refractive index structure function $C_{n}^{2}\left(1.2 \times 10^{-12} \mathrm{~m}^{-2 / a}\right)$, and lowest beam waist $w_{O R}=0.018 \mu \mathrm{rad}$ 


\section{International Journal of Science and Research (IJSR) \\ ISSN (Online): 2319-7064}

Index Copernicus Value (2013): 6.14 | Impact Factor (2015): 6.391

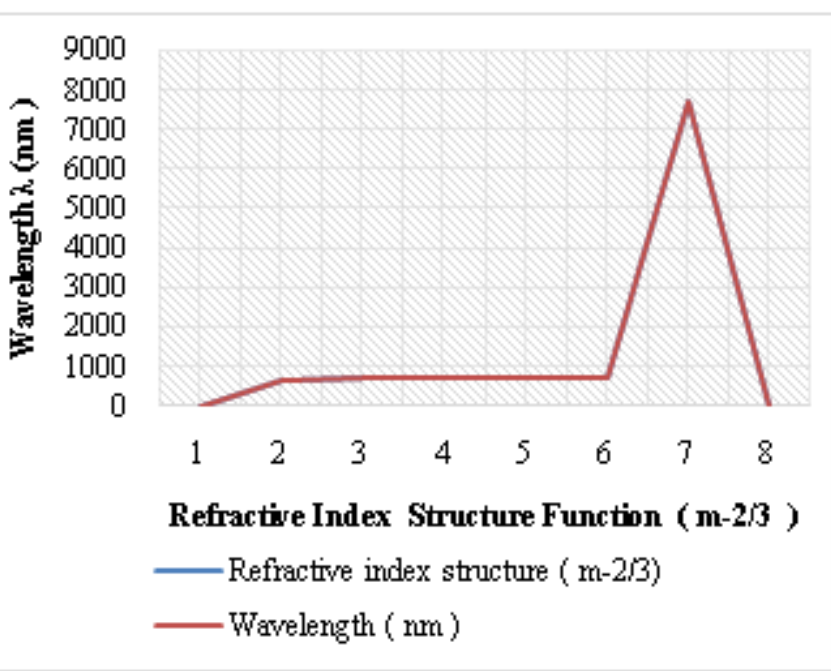

Figure 15: Relation between refractive indexstructure $C_{n}^{2}\left(m^{-\frac{2}{3}}\right)$ and wavelength $\lambda(n m)$

From figure (15), we note that at a maximum wavelength $(7715 \mathrm{~nm})$ occur the weak turbulence $\left(16 \times 10^{-21} \mathrm{~m}^{-2 / \mathrm{a}}\right)$ at a temperature $\left(424 \mathrm{k}^{\circ}\right)$. While the strong turbulence occurs ata wavelength equal $(716 \mathrm{~nm})$, at a temperature $\left(386 \mathrm{k}^{\circ}\right)$.

\section{Conclusion}

When the laser beam passing in a certain medium, (the medium either be a pure or it to be turbulent ), the amount of the propagation characteristic of the beam $\mathrm{M}^{2}$ represents indicator of how it influenced by the medium. This is an evidence of the extent of retaining beam analogue form (Gaussian function). The results confirmed that the laser beam passing through the non-homogeneous (random movement of molecules medium), the beam does not maintain the form analogue, where $M^{2}$ be less than one. The temperature that generated a turbulence model along pathway of the laser beam propagation, it found independently relative to other parameters that deviation from their values that resulted by influence of heat. This study and other in this area, is very useful to improving the performance of the systems such as: laser/beam communications systems, directed energy, Lidarthe refractive index structure, atmospheric coherence diameter, Gaussian function. the refractive index structure, atmospheric coherence diameter, Gaussian function.the refractive index structure, atmospheric coherence diameter, Gaussian function.the refractive index structure, atmospheric coherence diameter, Gaussian function., remote sensing, ect.

\section{References}

[1] Beer, and Tom (1975) "The aerospace environment ". Taylor \& Francis Ltd. pp. 26. ISBN 9780851090214 “.

[2] Strohbehn J.W., ( 1978 ), “ Laser beam propagation in the atmosphere ", Springer, Berlin,Heidelberg.

[3] Ishimaru A. (1981), " Theory of optical propagation in the atmosphere "ce , Opt.Eng.20.doi: $10.1117 / 12.7972665$.

[4] Shaik K.S., (1988 ), “ Atmospheric Propagation Effects relevant to Optical Communication ", The telecommunications and data acquisition progress report
42-94, ( E.C Posner, Ed ), NASA/Jet propulsion Laboratory, Pasadena, CA,pp.180-200

[5] Magee E.P., ( 1993 ), “ Characterization of Laboratory generated turbulence “, M.Sc. thesis. Air Force Institute of Technology, United States, pp 137.

[6] Tatarskii V I ( 1961 ), “ Wave Propagation in a Turbulent Medium. Translated from Russian by R.S. Silverman, McGraw-Hill, New-York, USA, 285pp.

[7] Baak T., (1969 )., "Thermal coefficient of refractive index of optical glasses “. J. Opt. Soc. Amer. 59, 851857.

[8] Ojo J. S., M. O. Ajewole and S. K. Sarkar, ( 2008 )., “ Rain rate and rain attenuation prediction for satellite communication in $\mathrm{Ku}$ and $\mathrm{Ka}$ bands over Nigeria ". Progress in Electrodynamics Research B 5, 207-223.

[9] Titterton D. H., ( 2005 )., "Development of infrared countermeasure technology and systems “. In: Midinfrared semiconductor optoelectronics (A. Krier, Ed.). Springer, pp. 635-671.

[10] Shin R. T. and J. A. Kong., ( 1989 ),. “ Radiative transfer theory for active remote sensing of two-layer random medium "., Progress in Electromagnetics Research 1, 359-417.

[11] Weichel H., ( 1990 ).," Laser beam propagation in the atmosphere ". SPIE, Washington, $98 \mathrm{pp}$.

[12] Ibrahim A. T., ( 2007 )., " Using microwave energy to treat tumors ". Progress in Electromagnetic Research B 3, 1-27.

[13] Chatterjee R. M. and H. A. M. Fathi,( 2014 ). "Investigation of profiled beam propagation through a turbulent layer and temporal statistics of diffracted output for a modified von Karman phase screen". Proc. SPIE 8971, doi:10.1117/12.2033442.

[14] Berman G. P., A. A. Chumak and V. N. Gorshkov, ( 2007 ). "Beam wandering in the atmosphere: The effect of partial coherence". Phys. Rev. E 76, doi:10.1103/PhysRevE.76.056606.

[15] Lonappen A., G. Bindu, V. Thomas, J. Jacob, C. Rajasekaran and K. T. Mathew, (2007). "Diagnosis of diabetes mellitus using microwaves". $J$. Electromagnet. Wave. 21, 1393-1401, doi:10.1163/156939307783239429. 